\title{
Contents of Volume 45 / Inhalt von Jahrgang 45 Sommaire du volume 45
}

Erwin H. Ackerknecht, Another Minority's Role in Medicine:

The Armenians

Erwin H. Ackerknecht, Cuvier and Medicine 309

- Sir Benjamin Ward Richardson and the Jews 323

- The Handicap of the Great Father 541

Carl Arbenz, Salvinavia, eine Wörtschöpfung des Naturforschers Chamisso 99

Gaetano Benedetti, Friedrich Nietzsche - ein Ahnherr der Psychoanalyse? 11

Marcel H. Bickel, The Development of Sulfonamides (1932-1938) as a Focal Point in the History of Chemotherapy $\quad 67$

Karin Biefel und Wolfgang Pirsig, Tracheotomien vor $1800 \quad 521$

U.B.Birchler-Argyros, Die Quellen zum Kral-Spital in Konstantinopel 419

Thomas N. Bonner, Pioneering in Woman's Medical Education in the Swiss Universities 1864-1950 461

Paul F. Cranefield, Carl Ludwig and Emil du Bois-Reymond: A Study in Contrasts 271

Jean Jacques Dreifuss, Julien Barry et les «synapses neurosécrétoires» (1954-1973) 87

- Charles Chossat (1796-1875), physiologiste, médecin et homme politique genevois 239

Hubert Fischer, Ärztliche Versorgung, sanitäre Verhältnisse und Humanversuche im Frauenkonzentrationslager Ravensbrück

Klaus-Dietrich Fischer, Das Gesundheitsgedicht des Burkhard von Horneck $(† 1522)$

J.Gasser, J. M. Charcot et la découverte des localisations motrices chez l'homme 501

Alfred Grafe, Die sogenannten Kochschen Postulate 411

Daniel Heinrich, Dr. med.Charlot Strasser (1884-1950) 483

Othmar Keel, Les rapports entre médecine et chirurgie dans la grande école anglaise de William et John Hunter

Georgette Legée, La physiologie française pendant la première moitié du XIX $X^{e}$ siècle. Ses rapports avec la physiologie suisse et allemande

William Arthur Liebi, Die chemische Behandlung physiologischer Probleme 201

Nikolaus Mani, Physiologische Konzepte von Galen bis Haller 165

Clemens Moser, Die Eisenbibliothek $\quad 561$

Paul Müller, Gabriel Gustav Valentin, Pionier der Berner Physiologie 191

Michael Quick, Die Lehre vom «Alpenstich» in den nosographischen Untersuchungen von Guggenbühl bis Sticker

Guy Saudan, La physiologie à la Haute-Ecole de Lausanne: le premier demi-siècle (1881-1932)

Gottfried Schramm, Ein Weihnachtsbrief Prof. Dr. Alexander Tschirchs, Bern, an seinen Bruder Prof. Dr. Otto Tschirch, Brandenburg a.d. Havel (1911)

Joseph Schürmann, Medizinisches aus Luzerner Sterbebüchern $\quad 543$

Antoinette Stettler, Sensation und Sensibilität 
Fritz Strauss, Kurzgeschichte der Placentarforschung

Hans H. Walser, Zum Hinschied von Erwin H. Ackerknecht

Volker Zimmermann, Zwischen Empirie und Magie: Die mittelalterliche

Frakturbehandlung durch die Laienpraktiker

Hansjürg Zumstein, Die Diskussion um die Euthanasie in Frankreich und der Schweiz vor dem Zweiten Weltkrieg

Book Reviews / Buchbesprechungen/Critiques de livres

Academiae Analecta, Bijdragen door R. Halleux, A.C.Debus, C. Meinel, H. A. M.

Snelders, A. Félix, C. Vanaempel, A. Bruylants, H. Deelstra (Daems)

Acta Medicae Historiae, Festschrift für Loris Permuda (Schramm)

148

Mechthild Amberger-Lahrmann und Dietrich Schmähl (Ed.), Gifte. Geschichte der Toxikologie (Ledermann)

Agnes Arber, Herbals. Their Origin and Evolution. History of Botany $1470-1670$ (Schmutz)

Réjane Bernier, Au sources de la biologie, Tome II (Mayer)

Die Werke von Daniel Bernoulli, Bd.3: Mechanik. Bearbeitet und kommentiert von

D. Speiser, A. de Baenst-Vandenbroucke, J.L. Pietenpol, P. Radelet-de Grave. Mit

Beiträgen von H.Straub (Neuenschwander)

Detlef Berthelsen, Alltag bei Familie Freud. Die Erinnerungen der Paula Fichtl

(C. Haffter)

H. Bocker und H.Thrum, Antibiotika - woher, wofür? (Bickel)

Lucien Braun, Paracelsus. Alchimist - Chemiker-Erneuerer der Heilkunde (Daems)

J. H. Cassecoy, The New Age of Health Laboratories 1885-1915 (Ackerknecht)

Ronald W. Clark, The Life of Ernst Chain (Bickel)

William Coleman, Yellow Fever in de North. The Methods of Early Epidemiology

(Ackerknecht)

Dietrich von Engelhardt, Thomas Henkelmann, Annette Krämer, Florenz und die

Toskana. Eine Reise in die Vergangenheit von Medizin und Wissenschaft (Quick)

Marianne Engesser, Der «Liber Servitoris» des Abulkasis (936-1013) (Daems)

Irene Fellmann, Das Aqrābādīn al-Qalānisī. Quellenkritische und begriffsanalytische Untersuchungen zur arabisch-pharmazeutischen Literatur (Daems)

Paul Feyerabend, Christian Thomas (Ed.), Leben mit den Acht Todsünden der Menschheit? Eine aktuelle Diskussion an der ETH zu den Thesen von Konrad Lorenz (Stettler)

Markus Fierz, Naturwissenschaft und Geschichte (Rieppel)

Uarda Frutiger, Ärztin im Orient, auch wenn's dem Sultan nicht gefält. Josephina

Th.Zürcher (1866-1932) (Stettler)

Danielle Gourevitch, Le triangle hippocratique dans le monde gréco-romain-le malade, sa maladie et son médecin (Koelbing)

Angela Graf-Nold, Der Fall Hermine Hug-Hellmuth. Eine Geschichte der frühen Kinderpsychoanalyse (C. Haffter)

Hermann Grensemann, Knidische Medizin Teil II (Schramm)

Otto Joachim Grüsser, Justinus Kerner, Arzt - Poet - Geisterseher (Ackerknecht) 
A. Rupert Hall, B. A. Bembridge, Physic and Philantrophy. A History of the Wellcome Trust 1936-1986 (Ackerknecht)

Bettina Haupt, Deutschsprachige Chemielehrbücher (1775-1850) (Ledermann)

Philipp Hauser, Atlas Epidemiografico del Colera de 1885 en Espagna (1887) (Ackerknecht)

Wolfgang-Hagen Hein, Alexander von Humboldt und die Pharmazie (Schramm) 572

Marie Gabrielle Hohenlohe, Die vielen Gesichter des Wahns (Müller)

R. Hooykaas, G.J.Rheticus' Treatise on Holy Scripture and the Motion of the Earth with translation, annotations, commentary and additional chapters of RamusRheticus and the development of the problems before 1650 (Daems)

Johann Friedrich Horner (1831-1886), Der Begründer der Schweizer Augenheilkunde in seiner Autobiographie (Bernoulli)

Franz Huter, Hieronymus Leopold Bacchettoni (Sigron)

Wolfgang Ilg, Die Regensburgische Botanische Gesellschaft. Ihre Entstehung, Entwicklung und Bedeutung, dargestellt anhand des Gesellschaftsarchives (Daems)

Serge Jagailloux, La médicalisation de l'Egypte au XIX $X^{e}$ siècle (1798-1918) (Mayer)

Dieter Jetter, Santiago, Toledo, Granada, drei spanische Kreuzhallenspitäler und ihr Nachhall in aller Welt (Schramm)

Engelbert Kaempfer, Phoenix Persicus. Die Geschichte der Dattelpalme (Dietlinde Goltz)

R. Kanigel, Apprentice to Genius. The Making of a Scientific Dynasty (Bickel)

Ernst Klee, Euthanasie im NS-Staat. Die «Vernichtung lebensunwerten Lebens» (C. Scharfetter)

Ingrid Klimaschewski-Bock, Die 'Distinction sexta' des Antidotarium Mesuë in der Druckfassung Venedig 1561 (Sirupe und Robub) (Daems)

Fritz Krafft (Herausgeber), Große Natuwissenschaftler (C. Haffter)

Helge Kragh, An Introduction to the Historiography of Science (Rieppel)

Gunhild Kübler, Geprüfte Liebe (C. Haffter)

Kirstin Landgraf-Brunner, Die Auseinandersetzungen zwischen Apothekern und den gesetzlichen Krankenkassen von Beginn der gesetzlichen Krankenversicherung an (Schramm)

Justus von Liebig und Emil Erlenmeyer in ihren Briefen von 1861-1872. Herausgegeben von Emil Heuser (Druey)

- und August Wilhelm Hofmann in ihren Briefen. Nachträge 1845-1869. Herausgegeben von Emil Heuser und Regine Zott (Druey)

H. Loeffel, Blaise Pascal, 1623-1662. Vita Mathematica, Bd.2 (Neuenschwander)

J. M. Lopez Pinero, El grabado en la ciencia Hispanica. Consejo superior de investigaciones cientificas (Ackerknecht)

- El Arte de navigar en la España del Renacimiento (Ackerknecht)

- M. J. Baguena Carvellera, J. L. Barona, J. L. Fresquet Febrer, M. L. Lopez Tirada, J. Pardo Tomas, V.L. Salvert Fabiani, Las ciencias Medicas basicas en la Valencia del siglo XIX (Ackerknecht)

Hilary Marland, Medecine and Society in Wakefield and Huddersfield 1780-1870 (Stettler) 
Markus Mattmüller, Bevölkerungsgeschichte der Schweiz, Teil I: Die frühe Neuzeit, 1500-1700, 2 Bände (Antoinette Stettler)

Christoph Mörgeli, Dr.med.Johannes Hegetschweiler (1789-1839) (Schaffner)

Henry M. Mollaret/Jacqueline Brossolet, Alexandre Yersin. Der Mann, der die Pest besiegte (Quick)

Eberhard Neumann/Redlin von Meding, Der Pathologe Ernst Neumann und sein Beitrag zur Hämatologie im 19. Jahrhundert (Ackerknecht)

Harald Nielsen, Medicaments used in the treatment of Eye Diseases in Egypt, the countries of the Near East, India and China in antiquity (Bernoulli)

Theophrast von Hohenheim genannt Paracelcus, Theologische und religionsphilosophische Schriften, Band III (Daems)

Bernhard Pauleikhoff, Ideologie und Mord (C. Scharfetter)

Felix Platter, Beschreibung der Stadt Basel 1610 und Pestbericht 1610/11 (M. L. Portmann)

Helmut Karl Reusch, Zur Geschichte der Lebensmittelüberwachung im Großherzogtum Baden und seinen Nachfolgeterritorien (1806-1954) (Schramm)

Charles E. Rosenberg: The Rise of America's Hospital System (Ackerknecht)

Detlef Rüster, Alte Chirurgie. Legende und Wirklichkeit (Sigron)

Guy Saudan (Ed.), L'Eveil médical vaudois (1750-1850) (Mayer)

Hansjörg Schneble, Krankheit der ungezählten Namen: Epilepsie anhand ihrer Benennungen vom Alterum bis zur Gegenwart (Ledermann)

Gottfried Schramm, Zur Geschichte der subkutanen Injektionen und Injektabilia in der zweiten Hälfte des 19. Jahrhunderts mit besonderer Berücksichtigung der Quecksilbertherapie (Daems)

Heinrich Otto Schröder, Publius Aelius Aristides. Heilige Berichte (Mudry)

Nancy G. Siraisi, Avicenna in Renaissance Italy. The 'Canon' and medical teaching in Italien universities after 1500 (Keil)

Brigitte Thanner, Hans Konrad Schmutz, Armin Geus, Johann Rudolf Schellenberg. Der Künstler und die naturwissenschaftliche Illustration im 18. Jahrhundert (H. Haffter)

Jacques Tremblay (Ed.), Les savants genevois dans l'Europe intellectuelle du XVII ${ }^{e}$ au milieu du XIX ${ }^{e}$ siècle (Dreifuss)

Richard J.Trudeau, The Non-Euclidean Revolution (Neuenschwander)

Arnold Uebelhart, Richard Scherb (1880-1995), Orthopäde und Muskelphysiologe (C. Haffter)

Michael Valentin, François Broussais 1772-1838. L'Empereur de la Médecine (Ackerknecht)

Manfred Vasold, Rudolf Virchow. Der große Arzt und Politiker (Ackerknecht)

Peter Voswinkel, 50 Jahre Deutsche Gesellschaft für Hämatologie und Onkologie (Ackerknecht)

Lorenz Welker, Das «Iatromathematische Corpus». Untersuchungen zu einem alemannischen astrologisch-medizinischen Kompendium des Spätmittelalters (C. Haffter) 
Stefan Winkle, Die heimlichen Spinozisten in Altona und der Spionzastreit (Ackerknecht)

Jörg Henning Wolf (Ed.), Aussatz, Lepra, Hansenkrankheit. Ein Menschheitsproblem im Wandel, Teil 2 (Antoinette Stettler)

K.G.Zysk, Religious Healing in the Veda (C.Scharfetter)

Buchanzeigen/Book Notices

Notes / Mitteilungen/Informations

158,589

Volume 45: Contents/Inhalt/Sommaire 